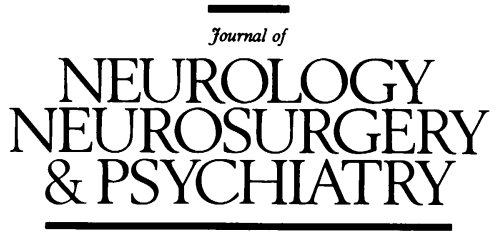

\title{
Editorial
}

\section{Management of primary malignant brain tumours}

Anaplastic astrocytoma and glioblastoma multiforme comprise the largest proportion of malignant supratentorial glioma. Most clinical and research papers considering aspects of either malignant glioma biology or treatment, contain, within the opening paragraph, a conditional statement similar to that first used by Bailey and Cushing in their 1926 monograph on brain tumours. ${ }^{1}$ The statement usually reads "despite recent advances in neuroimaging, microneurosurgery, radiotherapy, and chemotherapy malignant gliomas remain incurable". This group of neoplasms of the nervous system is the third commonest cause of cancer related death in young and middle aged adults. ${ }^{23}$ Perhaps even more depressing is that despite advances in investigative and therapeutic technology, a plethora of novel therapeutic approaches, and an increasing knowledge about their biology there has also been no major advance in the life expectancy of patients afflicted with these neoplasms.

Despite the highly variable quality of an increasingly voluminous literature on brain tumours much has been learnt about optimising management of patients with malignant gliomas. ${ }^{24-7}$ Transferring this information into good practice is important to avoid some of the potential pitfalls of therapeutic nihilism. Conversely, in many patients the omission of active treatments may be less detrimental than problems related to futile intensive therapies. Optimal management can range from using appropriate palliative strategies by a conscientious neuroclinician based in a general hospital to using novel, but unproved, treatments in the setting of a multidisciplinary cancer centre. What, therefore, should determine and influence decision making in the management of patients thought to have a malignant glioma?

Towards a diagnosis: diagnostic neuroradiology

The increasingly widespread use of MRI for neuroradiological diagnosis has enabled identification and diagnosis of focal lesions of the brain often when such lesions would not be visualised with CT. From the surgical perspective this has been particularly useful for the investigation of patients with no clinical abnormality but a history of adult onset seizures. ${ }^{8}$ A proportion of these patients with apparently innocuous focal lesions, as well as many patients diagnosed by CT to have low grade astrocytomas, will harbour an anaplastic astrocytoma when histological diagnosis is obtained. ${ }^{9}$ The difference between low grade astrocytoma and anaplastic astrocytoma is important as management strategies are different. ${ }^{10}$ Most malignant gliomas are, at the time of presentation with focal symptomatology, detectable by CT. Gadolinium enhanced MRI does, however, often show either a more extensive or multifocal lesion when compared with CT. Patients with malignant gliomas can present with quite variable symptomatology, ${ }^{11}$ and such neoplasms may also radiologically mimick the gamut of brain neuropathologies. ${ }^{12} \mathrm{~A}$ high index of suspicion must therefore be maintained to avoid the albeit low risk $<4 \%$ of misdiagnosising a benign non-neoplastic lesion as malignant glioma. ${ }^{12}$

In some patients tissue confirmation of diagnosis of malignant glioma may not be appropriate. This cohort includes patients in whom the history is of a progressive neurological deficit, neuroradiological studies show characteristic features suggestive of a glioblastoma, and clinical examination discloses major neurological or cognitive deficits and disability. Although most such patients will be elderly, biological age and both the patient's and their family's opinions must be considered and reasons for a non-interventional management approach explained. The rationale for this nihilistic but pragmatic approach is based on the very poor prognosis, both for life expectancy and functional improvement even after multimodality therapy, associated with glioblastoma in the elderly patient ( $>60$ years) who presents with poor functional status. ${ }^{24512}$ In this cohort judicious use of glucocorticoid treatment will optimise neurological function. Good primary medical practice and the use of home nursing and hospice care provide best management for most of these patients. The facility for additional neurooncological follow up should, however, be retained as an option as even without treatment some of these patients survive longer than expected, and are at risk of major steroid related complications and even misdiagnosis. ${ }^{12-14}$

\section{Making the diagnosis: surgical approaches}

Perhaps one of the major recent advances in the management of patients with malignant brain tumours has been the decrease in morbidity and mortality associated with both biopsy and major cytoreductive surgery. Before the advent of image-guided stereotactic biopsy techniques diagnostic surgery was often associated with unacceptable levels of morbidity, mortality, and failure to make a definite pathological diagnosis. ${ }^{15}$ Nowadays with MR or CT guidance systems the morbidity of stereotactic biopsy 
of lesions anywhere in the brain, even in the frail and elderly, is usually $<5 \%$ and the mortality $<1 \%$. ${ }^{1617}$ In many cases the 30 day morbidity associated with biopsy is either transient or related to the rapid progression of the primary disease process. The importance of confirmation of the diagnosis of malignant glioma has been emphasised in several papers particularly when there may be neuroradiological uncertainty between diagnoses of malignant glioma, metastatic lesion, abscess, inflammatory lesion, or other contrast enhancing neuropathological processes. ${ }^{1217}$ An experienced neuroradiologist, provided with appropriate clinical information, will correctly diagnose $95 \%$ of CT imaged malignant brain tumours. ${ }^{12}$ Therefore in many cases biopsy confirmation of a diagnosis of malignant glioma could be argued to have added little to patient management. Tissue diagno'sis, however, where appropriately indicated, facilitates making decisions to employ interventional therapies as patients with anaplastic astrocytoma and anaplastic oligodendroglioma respond significantly better to interventional treatments than those with glioblastoma. ${ }^{25618-21}$ In both middle aged and elderly patients, tissue diagnosis allows realistic discussion with the patient and their immediate families about both prognosis and subsequent management decisions.

Whether cytoreductive surgery is helpful in malignant glioma depends on the outcome measured. Certainly signs and symptoms related to raised intracranial pressure can be relieved, seizure frequency and intensity reduced, and many focal neurological deficits improved. ${ }^{2}$ 51122-24 Requirements for corticosteroid treatment, which can be a cause of significant morbidity in patients with brain tumours, ${ }^{14}$ are also either considerably reduced or abolished postoperatively. With the technical facilities and adjuncts that are now available, such as three dimensional and multiplanar reconstructions of MRI, high quality operating microscopes, precision microinstrumentation, frame based or frameless stereotaxic craniotomy and lesion localisation techniques, ${ }^{25}$ and awake craniotomy with cortical mapping techniques ${ }^{26}$ cytoreductive surgery can be performed safely and effectively. The morbidity associated with such procedures will be determined by the experience and skill of the surgical neuro-oncologist. As there is a fine balance between improving and worsening neurological function with cytoreductive surgery it is imperative, as these patients have an incurable disease with a poor natural history, that iatrogenic disability is minimised.

The contribution of cytoreductive surgery to improving median survival times is controversial. ${ }^{25}$ In some studies extensive surgical resection of malignant glial tumours has been shown to be an independent prognostic variable ${ }^{42}$ whereas in others it is not a significant factor. ${ }^{27}{ }^{28}$ Unfortunately much of the medical literature on the role of surgery for malignant glioma is unsatisfactory as series are often retrospective, not randomised between biopsy and major resection, not stratified for the important independent prognostic variables such as age, performance, and neuropathological subgroup, and often contain inadequate numbers for appropriate statistical analysis. ${ }^{2}$ None the less any benefits of cytoreductive surgery probably become less with both increasing age of the patient and increasing tumour malignancy. ${ }^{2} 13$ The limited survival benefit bestowed by even the most exacting cytoreductive operation for malignant glioma, and particularly glioblastoma, is related to the invasive and infiltrative potential of malignant glioma. ${ }^{7}$ These properties seem related to the production of plasminogen activators, matrix metalloproteinases, and the secretion of certain growth factors, all of which enhance malignant cell migration through the extracellular brain matrix. ${ }^{29}$ Generally peritumoral invasion extends for $2 \mathrm{~cm}$ beyond either the macroscopic tumour margin or the borders defined by enhanced CT. ${ }^{7}$ In most locations the likelihood of iatrogenic brain dysfunction prohibits excision of infiltrated peritumorous brain. Even when extensive peritumorous brain resection has been undertaken tumour recurrence at the margin of resection is inevitable. ${ }^{724}$

\section{Management after diagnosis: lessons from clinical trials}

External beam brain irradiation remains the principal treatment in the management of malignant gliomas. An optimal total dose of $60 \mathrm{~Gy}$ in 30 fractions is conventional. ${ }^{230}$ In many prospective studies, of mixed anaplastic astrocytoma and glioblastoma cohorts, such treatment will have contributed to a median survival time of between nine and 11 months. ${ }^{25}$ The addition of a radiosensitiser during radiotherapy has not significantly improved survival times ${ }^{31} 32$ and doses $>60$ Gy are associated with a high incidence of acute and short term neurological side affects and later cerebral radionecrosis with no benefit in either median or longer term survival. ${ }^{233}$

Since the 1970 s chemotherapy using a nitrosourea has also been widely used either as adjuvant therapy after radiotherapy or after glioma recurrence. ${ }^{25-720}$ Metaanalysis suggests a small survival advantage can be obtained with adjuvant chemotherapy. ${ }^{34}$ Recent studies suggest single agent nitrosourea therapy is as effective as multi agent therapy. ${ }^{6}$ Responses, in terms of median survival time and percentage of survivors at two years, to radiotherapy and chemotherapy, are better in patients with either an anaplastic oligodendroglioma ${ }^{19}$ or an anaplastic astrocytoma than those with glioblastomas. Review of data from different prospective, randomised clinical trials has shown that patients with anaplastic astrocytoma have median survival times from 36 months $^{21}$ to two years ${ }^{618}$ compared with a median 8.6 months and $10 \%$ two year survivors for patients with glioblastoma. ${ }^{61821}$ Other favourable prognostic variables are younger age, good performance status, and a previous history of seizures. ${ }^{24512}$ Using these factors predictions about survival can be made. ${ }^{4}$

Disease relapse after both radiotherapy and chemotherapy is invariably due to recurrence within $2 \mathrm{~cm}$ of the original lesion. ${ }^{2733}$ Why do these treatments, which theoretically should sterilise infiltrated peritumorous brain, not give better results? Studies of the radiobiology of malignant glioma cell lines in vitro show that they have an inherently high radioresistance, with a large capacity for DNA repair which can continue without major slowing of the cell cycling time (Ross G, personal communication). Failure of chemotherapy for malignant gliomas is probably related to both inherent tumour cell chemoresistance and failure of drug delivery. ${ }^{3}{ }^{35}$ The blood-brain barrier can limit transport of large molecular weight, polarised, non-lipophilic agents into peritumorous brain; however, in the tumour interstitium there is no comparable biophysical barrier. ${ }^{36}$ Many glioma cell lines are inherently resistant to doses of chemotherapeutic agents that may be attained in vivo and even non-conventional high dose therapy given with bone marrow rescue. ${ }^{3}$ Such resistance is related to drug efflux systems such as the multidrug resistance (mdr) gene, and the enzyme O6-alkyl-guanine-DNA alkyltransferase, which limits the toxicity of alkylating agents. ${ }^{337}$ A multiplicity of novel chemotherapeutic agents and drug regimes have been given in phase 2 and phase 3 studies to patients with relapsed malignant glioma. ${ }^{38}$ Despite some initial promise 
for some of these drugs nitrosoureas remain as effective as these novel agents. Therapeutic approaches designed to improve drug delivery to the tumour include supraophthalamic intracarotid arterial infusion, ${ }^{39}$ osmotic opening of the blood-brain barrier, and the use of implantable polyanhydride polymers impregnated with chemotherapeutic agents. ${ }^{40}$ Results are variable and require cautious interpretation.

Interstitial radiotherapy using stereotactically placed catheters after-loaded with radioactive seeds can contribute to prolongation of survival in selected patients with recurrent disease. ${ }^{2}{ }^{33}$ Interstitial brachytherapy is, however, associated with focal radionecrosis that often requires steroid treatment, or resection, or both. ${ }^{33}$ Clinical trials are currently evaluating the role of interstitial brachytherapy, as an adjunct to external beam radiotherapy in both the primary management and recurrence of malignant glioma. Other studies have considered the roles of hyperfractionated external beam radiation therapy treatment regimes, ${ }^{2}$ hyperthermia, ${ }^{33}$ and the role of stereotactic radiosurgery using either the gamma knife or a linear accelerator. Unfortunately, the preliminary results with stereotactic radiosurgery for malignant glioma are disappointing. ${ }^{41}$ None the less with continuing advances in brain imaging software for radiobiology, further novel therapeutic approaches will probably be undertaken in these fields and that of photodynamic therapy as new photosensitisers are identified. ${ }^{42}$

One of the newest approaches to treatment of brain tumours is the use of suicide genes, such as herpes simplex thymidine kinase (HS-tk) transfected into tumour cells using a retroviral vector. ${ }^{43}$ The transfected cells are rendered sensitive to the cytotoxic effects of gancyclovir. Although this paradigm has worked well in rodent models of brain tumour, ${ }^{44}$ the results of the initial clinical studies in humans have been disappointing (Oldfield E, personal communication). Further work is required to improve both the methods and efficiency of gene transfection of glioma cells and the selection of genes to be used. ${ }^{45}$ Whether these problems are surmountable or whether gene therapy for malignant gliomas will prove as disappointing as immunomodulative therapy ${ }^{16}$ and monoclonal antibody therapy ${ }^{47}$ awaits further studies.

Overall, when reviewing series of different management strategies in patients with malignant brain tumour, it is important to remember that these series report patients who are selected for the studies because of their good prognostic features. An audit of practice in south east Scotland, which is served by a dedicated neurooncology service, showed that only $40 \%$ of patients with malignant glioma ever receive either surgery or radiotherapy. Furthermore, there were often significant delays between diagnosis and treatment. Selection of a patient for surgery, or even neuro-oncological referral, depends on many factors including their clinical condition at the time of neuroradiological imaging, the interpretation of the neuroradiological report, the knowledge of the referring physician, the attitudes of the local oncological and neurosurgical services to interventional neuro-oncology, and the desires of the patients and their immediate families. Management of the patients not referred to a neurooncological service is as important as those undergoing intensive therapies. Access to written information, and time for explanantion of the disorder with the patient and their family and reasons for management decisions form an important part of the basic care of patients with malignant glioma. ${ }^{48}$ Because cure of malignant gliomas is often impossible therapeutic endeavours should be primarily aimed at improving the patient's quality of life. Quality of life issues are becoming increasingly recognised as impor- tant in patients with malignant gliomas. ${ }^{49}$ Although this is a difficult concept to assess studies using self report questionnaires assessing "well being" have identified freedom from depression, an active social life, energy, and fewer symptoms as being important. The commonly used Karnofsky performance scale was found to be heavily influenced by age and not related to "well being" even though patients had a high Karnofsky performance score. ${ }^{50}$ With the increasing demand for evidence based medicine it is likely that there will be an intensification of research in these areas and a long overdue expansion of support and caring facilities for patients with malignant brain tumours.

Department of Clinical Neuroscience,

IAN R WHITTLE

Western General Hospital,

Edinburgh EH4 $2 X U$, UK

1 Bailey P, Cushing H. A classification of the tumors of the glioma group on an istogenic basis with a correlated study of prognosis. Philadelphia: Lippincott, 1926.

2 Fine HA. The basis for current treatment recommendations for malignant gliomas. 7 Neurooncol 1994;20:111-20.

3 Petersdorf $\mathrm{SH}$ and Livingston RB. High dose chemotherapy, for the treatment of malignant brain tumours. F Neurooncol 1994;20:155-63.

4 MRC Brain Tumour Working Party. Prognostic factors for high grade gliomas: development of a prognostic index. F Neurooncol 1990;9:47-55.

5 Shapiro WR. Therapy of adult malignant brain tumors: what have the clinical trials taught us? Semin Oncol 1986;13:38-45.

6 Shapiro WR, Green SB, Burger PC, et al. Randomized trial of three chemotherapy regimens and two radiotherapy regimens in postoperative treatment of malignant glioma. F Neurosurg 1989;71:1-9.

7 Wilson CB. Glioblastoma: past, present, and future. Clin Neurosurg 1992;38:23-48.

8 Zentner J, Hufnagel A, Wolf HK et al. Surgical treatment of temporal lobe epilepsy: clinical radiological, and histopthological finding in 178 patients. 尹 Neurol Neurosurg Psychiatry 1995;58:666-73.

9 Chamberlain MC, Murovic JA, Levin VA. Absence of contrast enhancement on CT brain scans of patients with supratentorial malignant gliomas. Neurology 1988;38:1371-4

10 Cairncross JG, Lapierrre NJ. Low grade glioma: to treat or not to treat? Arch Neurol 1989;46:1238-9.

11 Whittle IR. Origins and management of peritumoural brain dysfunction. Neurosurgery Quarterley 1992;2:174-98.

12 Chocksey M S, Valentine A, Shawdon H. Computed tomography in the diagnosis of malignant brain tumours. Do all require biopsy? $\mathcal{f}$ Neurol Neurosurg Psychiatry 1989;52:821 - 25.

13 Whittle IR, Denholm SW, Gregor A. Management of patients aged over 60 years with supratentorial glioma:lessons from an audit. Surg Neurol 1991;36:106-11.

14 Dropcho EJ, Soong S. Steroid-induced weakness in patients with primary brain tumors. Neurology 1991;41:1235-39.

15 Hitchcock E, Sato FJ. Treatment of malignant gliomata. $f$ Neurosurg 1964;21:497-506.

16 Appuzzo ML, Sabshin JK. Computed tomographic guidance stereotaxis in the management of intracranial mass lesions. Neurosurgery in the managen $1983 ; 12: 77-85$.

17 Thomas DGT, Nouby RM. Experience in 300 cases of CT-directed stereotactic surgery for lesion biopsy and aspiration of hematoma. $\mathrm{Br}$ Neurosurg 1989;3:321-6.

18 Burger PC, Vogel FS, Green SB, et al. Glioblastoma multiforme and anaplastic astrocytoma. Cancer 1985;56:1106-11.

19 Cairncross G, Macdonald D, Ludwin S, et al. Chemotherapy for anaplastic oligodendroglioma. J Clin Oncol 1994;12:2013-21.

20 Gregor A, Rampling R, Aapro M, et al. Phase II study of tauromustine in malignant glioma. Eur 7 Cancer 1992;28A: 1959-62.

21 Nelson DF, Nelson JS, Davis DR et al. Survival and prognosis of patients with astrocytomas with atypical or anaplastic features. 7 Neurooncol 1985;3:99-103

22 Jeremic B, Grujicic D, Antunovic V, et al. Influence of extent of surgery and tumour location on treatment outcome of patients with glioblastoma multiforme treated with combined modality approach. $f$ Neurooncol 1994;21:177-85.

23 Ammirati $M$, Vick N, Liao Y, et al. Effect of the extent of surgical resection on survival and quality of life in patients with supratentoria tion on survival and quality of life in patients with supratentorial

24 Selby $R$. The surgical treatment of cerebral glioblastoma multiforme: an historical review. $\mathcal{F}$ Neurooncol 1994;18:175-82.

25 Sandeman DR, Patel N, Chandler C, et al. Advances in image directed neurosurgery: preliminary experience with the ISG viewing wand compared with the Lekesell G frame. Br $\mathcal{F}$ Neurosurg 1994;8:529-94

26 Black P McL, Ronner SF. Cortical mapping for defining the limits of tumour resection. Neurosurgery 1987;20:914-9.

27 Coffey RJ, Lunsford LD, Taylor FH. Surivival after stereotactic biopsy of malignant gliomas. Neurosurgery 1988;22:465-73.

28 European Organization for Research on Treatment on Cancer (EORTC) Brain Tumor Group. Evaluation of CCNU,VM 26 plus CCNU and procarbazine in supratentorial brain gliomas. Final evaluation of a randomized study. 7 Neurosurg $1981 ; 55 \cdot 27-31$.

29 Thorgeirsson UP, Lindsay CK, Cottam DW, Gomez DE. Tumour invasion, proteolysis and angiogenesis. F Neurooncol 1994;18:89-103.

MRC Brain Tumour Working Party. A Medical Research Council trial of two radiotherapy doses in the treatment of grade 3 and 4 astrocytoma. two radiotherapy doses in the

31 MRC Working Party on misonidazole in gliomas. A study of the effect of misonidazole in conjunction with radiotherapy for the treatment of grades 3 and 4 astrocytomas. Br $\mathcal{F}$ Radiol 1983;56:673-82 
32 Bleehen NM, Freedman LS, Stenning SP. A randomized study of CCNU with and without benznidazole in the treatment of recurrent grades 3 and 4 astrocytoma. Int $\mathcal{F}$ Radiat Oncol Biol Phys 1989;16:1077-8.

33 Sneed PK, Gutin PH. Interstitial radiation therapy of brain tumours. In: Morantz RA, Walsh JW, eds. Brain tumours, New York: Marcel Dekker Inc, 1994:79-92.

34 Stenning SP, Freedman LS, Bleehen NM. An overview of published results from randomized studies of nitrosoureas in primary high grade malignant glioma. Br f Cancer 1987;56:89-90.

35 Blasberg RG, Groothuis DR. Chemotherapy of brain tumors: physiological and pharmacokinetic considerations. Semin Oncol 1986;13:70-82.

36 Seitz RJ, Wechsler W. Immunohistochemical demonstration of serum proteins in human cerebral gliomas. Acta Neuropath (Berl) 1987:73. 145-52.

37 Sariban E, Kohn KW, Zlotogorski C et al. DNA cross-linking responses of human malignant glioma cell strains to chloroethylnitrosoureas, cisplatin and diazequone. Cancer Res 1987:47:3988-94

38 Taylor SA. ew agents in the treatment of primary brain tumours. $f$ Neurooncol 1994:20:141-53.

39 Loew F, Papavero L. The intra-arterial route of drug delivery in the chemotherapy of malignant brain tumour. Adva Tech Stand Neurosurg 1988:16:51-82

40 Brem H, Piantadosi S, Burger PC, et al. Placebo-controlled trial of safety and efficacy of intraoperative controlled delivery by biodegrable polymers of chemotherapy for recurrent gliomas. Lancet 1995;345:1008-12.
41 Masciopinto J, Levin AB, Melita MP, et al. Stereotactic radiosurgery for glioblastoma: a final report of 31 patients. $\mathcal{F}$ Neurosurg 1995;82:530-35.

42 Kaye A, Hill J S. Photodynamic therapy of brain tumours. Neurosurgery Quarterly 1992;1:223-45.

43 Oldfield EH, Ram Z, Culver KW, et al. Clinical protocols: gene therapy for the treatment of brain tumours using intratumoural transduction with the thymidine kinase gene and intravenous ganciclovir. Hum Gen Ther 1993;4:39-69.

44 Culver KW, Ram Z, Walbridge S, et al. In vivo gene transfer with retroviral vector-producing cells for the treatment of experimental brain tumours. Science 1992;256:1550-2.

45 Brown JY, Saleh M. Retroviruses and DNA: gene therapy for brain tumours. F Clin Neurosci 1995;2:16-23.

46 Jaeckie KA, Immunotherapy of malignant glioma. Semin Oncol 1994 21:49-59.

47 Nitta T, Sato K, Yagita $\mathrm{H}$, et al. Preliminary trial of specific targetting therapy against malignant glioma. Lancet 1990;335:368-76.

48 Whittle IR, Broadbent M, Boyd A, et al. Public perceptions of brain tumours in Scotland: the need for access to information. Scottish tumours in Scotland: the need

49 Aiken Robert D. Quality of life issues in patients with malignant gliomas. Semin Oncol 1994;21:273-5.

50 Mackworth N, Fobair P, Prados MD. Quality of life self reports from 200 brain tumour patients: comparisons with Karnofsky performance. Neurooncol 1992;14:243-53.

\section{NEUROLOGICAL STAMP}

\section{Willow}

Two thousand, four hundred years ago Hippocrates recommended chewing willow leaves for analgesia during childbirth and for postpartum fever. Pliny in Rome in the first century $\mathrm{AD}$ prescribed the bark of the poplar (which is also a member of the willow (salix) family) for sciatic pain.

In the 1830s salacin and its derivative salicylic acid were isolated from white willow and various other plants. In the 1870s, salicylic acid was synthesised. Felix Hoffman, a chemist at the Bayer Pharmaceuticals Company in Germany later produced a modified form of salicylic acid, the acetyl derivative, which was effective against fever and arthritic pain. Its antithrombotic properties were described in the 1940s. The name aspirin came from the Spiraea plant, one of the other sources of salicylic acid. The prefix $a$ was added to signify acetyl. Aspirin contains no willow derivatives and is entirely synthetic.

A row of willows is shown on a stamp issued in 1973 depicting Swedish landscapes (Stanley Gibbons 736, Scott 158).

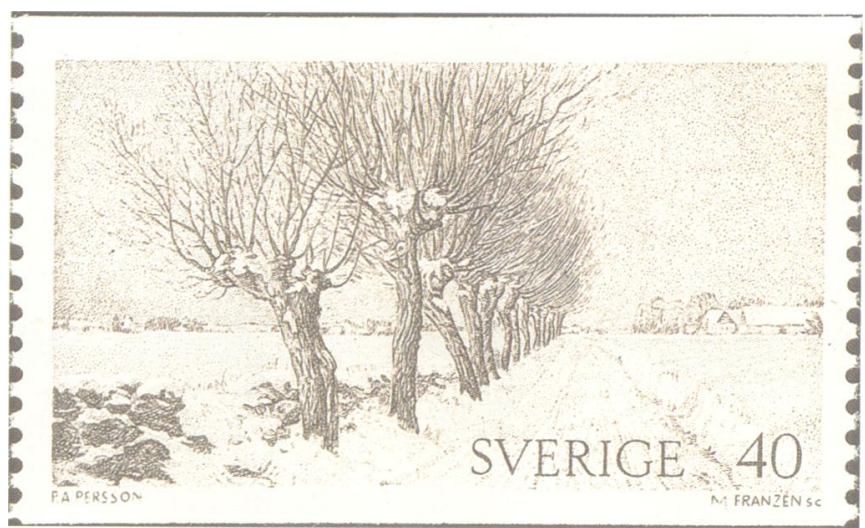

